\title{
Heat transfer mechanisms during short-pulse laser heating of two-layer composite thin films
}

\author{
M. A. Al-Nimr, M. Alkam, V. Arpaci
}

\begin{abstract}
Using a simple perturbation technique, an analytical investigation is presented for the heat transfer mechanisms during ultrafast laser heating of two-layer composite thin slabs from a microscopic point of view. The composite slab consists of two thin metal films which are in perfect thermal contact. The microscopic parabolic two-step model is adopted to describe the behavior of the composite slab. In the microscopic two-step model, the heating process is modeled by the deposition of radiation energy on electrons, the transport of energy by electrons, and the heating of the material lattice through electronphonon interactions. The proposed perturbation technique is used when the normalized temperature difference between the solid lattice and the electron gas is relatively a small perturbed quantity.
\end{abstract}

\section{List of symbols}

$C$ heat capacity, $\mathrm{J} / \mathrm{m}^{3} \mathrm{~K}$

$C_{\mathrm{R}}$ heat capacity ratio, $C_{2} / C_{1}$

$G$ electron-phonon coupling factor, $\mathrm{W} / \mathrm{m}^{3} \mathrm{~K}$

$G_{i j} \quad$ Green's function

$h_{1} \quad$ thickness of the first layer, $m$

$h_{2}$ total thickness of the two layers, $\mathrm{m}$

$k$ thermal conductivity, $\mathrm{W} / \mathrm{m} \mathrm{K}$

$k_{\mathrm{R}} \quad$ thermal conductivity ratio, $k_{2} / k_{1}$

$P_{\mathrm{el}}$ dimensionless heat source in the electron gas of the first layer, $Q_{\mathrm{e} 1} h_{2}^{2} / k_{\mathrm{e} 1} T_{\mathrm{i}}$

$P_{\mathrm{e} 2}$ dimensionless heat source in the electron gas of the second layer, $Q_{\mathrm{e} 1} h_{2}^{2} C_{\mathrm{e} 1} / k_{\mathrm{e} 1} T_{\mathrm{i}} C_{\mathrm{e} 2}$

$P_{11} \quad$ dimensionless heat source in the solid lattice of the first layer, $Q_{11} h_{2}^{2} C_{\mathrm{e} 1} / k_{\mathrm{e} 1} T_{\mathrm{i}} C_{\mathrm{l} 1}$
Received on 20 September 2000 / Published online: 29 November 2001

M. A. Al-Nimr $(\bowtie)$

Mechanical Engineering Department

University of Jordan

Amman, Jordan

E-mail: malnimr@just.edu.jo

M. Alkam

Mechanical Engineering Department

Jordan University of Science and Technology

Irbid, Jordan

V. Arpaci

Mechanical Engineering Department

University of Michigan

Ann Arbor, USA
$P_{12}$

$Q_{\mathrm{e}}$

$Q_{1}$

$r$

$R$

$t$

$t_{0}$

$t_{\mathrm{p}}$

$T$

$T_{\mathrm{i}}$

$z$

$Z$

\section{Greek symbols}

$\alpha \quad$ radiation absorption coefficient, $1 / \mathrm{m}$

$\delta \quad$ Dirac's delta function

$\Delta$ difference function

$\epsilon \quad$ dimensionless small parameter

$\theta$ dimensionless temperature, $\left(T-T_{\mathrm{i}}\right) / T_{\mathrm{i}}$

$\tau \quad$ dimensionless time, $t k_{\mathrm{e} 1} /\left(h_{2}^{2} C_{\mathrm{e} 1}\right)$

$\tau_{0} \quad$ dimensionless time at which the laser pulse is released, $t_{0} k_{\mathrm{e} 1} /\left(h_{2}^{2} C_{\mathrm{e} 1}\right)$

$\tau_{\mathrm{p}} \quad$ dimensionless laser pulse duration, $t_{\mathrm{p}} k_{\mathrm{e} 1} /\left(h_{2}^{2} C_{\mathrm{e} 1}\right)$

\begin{tabular}{ll}
\multicolumn{2}{l}{ Subscripts } \\
1 & first layer \\
2 & second layer \\
e & electron \\
i & initial \\
1 & lattice
\end{tabular}

\section{Introduction}

Research of high-rate heating on thin film structures has rapidly grown in recent years because of the advancement of short-pulse laser technologies and their applications to modern microfabrication technologies $[1,2]$. To date, the laser pulse can be shortened to the range of femtoseconds $\left(10^{-15} \mathrm{~s}\right)[3,4]$ making controls of the penetration depth and the processing time of the material more effective and accurate.

Associated with shortening the response time, the nonequilibrium thermodynamic transition and the microscopic effects in the energy exchange are two important issues to be faced. The microscopic mechanisms of energy deposition become important when the heating process is 
very fast. Non equilibrium electron and lattice temperatures in metals are proposed by Kaganov et al. [5] and confirmed experimentally by Eesley [6].

In the literature, there are basically four models that describe the mechanism of energy transport during shortpulse laser heating. The first model is the parabolic onestep model, which is based on the classical Fourier conduction law. This model assumes that solid lattice and electron gas are in local thermal equilibrium and that heat flux merges instantaneously when temperature gradients exist. The second model is the hyperbolic one-step model $[7,8]$ which is first postulated for gases by Maxwell [9]. In this model, it is assumed that both lattice and electron gas are in local thermal equilibrium but the heat flux and the temperature gradient are non-local in time. This implies that heat flux lags the temperature gradient by the thermal relaxation time. The third and fourth models are the parabolic two-step and the hyperbolic two-step models [10-16]. In these two models, it is assumed that solid lattice has different temperature than electron gas and the difference between these two temperatures depends on the coupling factor between both domains. However, in the parabolic two-step model, it is assumed that both heat flux and temperature gradients are local in time. This implies that heat flux in the electron gas (or in the solid lattice in applications involving semiconductors) merges instantaneously as soon as temperature gradients in the electron gas (or in the solid lattice) exist. On the other hand, the hyperbolic two step model, which is proposed by Qiu and Tien [11], assumes that heat flux and temperature gradient are non-local in time. In other words, the heat flux in the electron gas (or in the solid lattice) lags the temperature gradient by a thermal relaxation time.

In the literature, intensive research has been conducted using the above mentioned four models. To the best of the authors' knowledge all the research in the literature consider the interaction of high-rate heating source with a single thin metal film from macroscopic and microscopic points of views or the interaction of high-rate heating source with a two-layer composite thin slab from a macroscopic point of view. In the present work, it is intended to model the heat transfer characteristics of a two-layer composite thin slab upon the application of a high-rate heating source from a microscopic point of view. Using a simple perturbation technique, an analytical investigation is presented for the heat transfer mechanisms during ultrafast laser heating of the two-layer composite thin slabs. The two-layer composite slab consists of two thin metal films which are in perfect thermal contact. The proposed perturbation technique is used when the normalized temperature difference between the solid lattice and the electron gas is relatively a small perturbed quantity.

\section{2}

\section{Analysis}

Consider the interaction of a high-rate heating laser beam with a two-layer slab that consists of two thin films in perfect thermal contact. Using the dimensionless parameters defined in the nomenclature, the governing equations in their dimensionless form are given as:

$$
\begin{aligned}
& \frac{\partial \theta_{\mathrm{e} 1}}{\partial \tau}=\frac{\partial^{2} \theta_{\mathrm{e} 1}}{\partial Z^{2}}-H_{1}\left(\theta_{\mathrm{e} 1}-\theta_{\mathrm{l} 1}\right)+F_{1} P_{\mathrm{e} 1} \\
& \frac{\partial \theta_{\mathrm{l} 1}}{\partial \tau}=H_{2} \frac{\partial^{2} \theta_{\mathrm{l1}}}{\partial Z^{2}}+H_{3}\left(\theta_{\mathrm{e} 1}-\theta_{\mathrm{l} 1}\right)+\left(1-F_{1}\right) P_{11} \\
& \frac{\partial \theta_{\mathrm{e} 2}}{\partial \tau}=H_{4} \frac{\partial^{2} \theta_{\mathrm{e} 2}}{\partial Z^{2}}-H_{5}\left(\theta_{\mathrm{e} 2}-\theta_{\mathrm{l} 2}\right)+F_{2} P_{\mathrm{e} 2} \\
& \frac{\partial \theta_{\mathrm{l} 2}}{\partial \tau}=H_{6} \frac{\partial^{2} \theta_{\mathrm{l}}}{\partial Z^{2}}+H_{7}\left(\theta_{\mathrm{e} 2}-\theta_{\mathrm{l} 2}\right)+\left(1-F_{2}\right) P_{\mathrm{l} 2}
\end{aligned}
$$

where

$$
\begin{aligned}
& H_{1}=\frac{G_{1} h_{2}^{2}}{k_{\mathrm{e} 1}}, \quad H_{2}=\frac{k_{\mathrm{l} 1} C_{\mathrm{e} 1}}{k_{\mathrm{e} 1} C_{\mathrm{l} 1}}, \quad H_{3}=\frac{h_{2}^{2} C_{\mathrm{e} 1} G_{1}}{k_{\mathrm{e} 1} C_{\mathrm{l} 1}}, \\
& H_{4}=\frac{k_{\mathrm{eR}}}{C_{\mathrm{eR}}}, \quad H_{5}=\frac{G_{2} h_{2}^{2}}{k_{\mathrm{e} 1} C_{\mathrm{eR}}}, \quad H_{6}=\frac{C_{\mathrm{e} 1} k_{\mathrm{l} 2}}{C_{\mathrm{l} 2} k_{\mathrm{e} 1}}, \\
& H_{7}=\frac{G_{2} h_{2}^{2} C_{\mathrm{e} 1}}{k_{\mathrm{e} 1} C_{\mathrm{l} 2}}, \\
& P_{\mathrm{e} 1}=\frac{Q_{\mathrm{e} 1} h_{2}^{2}}{k_{\mathrm{e} 1} T_{\mathrm{i}}}, \quad P_{\mathrm{l} 1}=\frac{Q_{\mathrm{l} 1} h_{2}^{2} C_{\mathrm{e} 1}}{k_{\mathrm{e} 1} T_{\mathrm{i}} C_{\mathrm{l} 1}}, \\
& P_{\mathrm{e} 2}=\frac{Q_{\mathrm{e} 2} h_{2}^{2} C_{\mathrm{e} 1}}{k_{\mathrm{e} 1} T_{\mathrm{i}} C_{\mathrm{e} 2}}, \quad P_{\mathrm{l} 2}=\frac{Q_{\mathrm{l} 2} h_{2}^{2} C_{\mathrm{e} 1}}{k_{\mathrm{e} 1} T_{\mathrm{i}} C_{\mathrm{l} 2}} .
\end{aligned}
$$

where $F_{1}$ and $F_{2}$ represent the fraction of absorbed incident radiation by the electron gas in the first and second layers, respectively, and as a result, $1-F_{1}$ and $1-F_{2}$ represent the fraction of energy absorbed by the solid lattice in the first and second layers, respectively.

Equations (1-4) assume the following initial and boundary conditions:

$$
\begin{aligned}
& \theta_{\mathrm{e} 1}(0, Z)=\theta_{\mathrm{e} 2}(0, Z)=\theta_{\mathrm{l} 1}(0, Z)=\theta_{\mathrm{l} 2}(0, Z)=0 \\
& \frac{\partial \theta_{\mathrm{e} 1}}{\partial Z}(\tau, 0)=\frac{\partial \theta_{\mathrm{l} 1}}{\partial Z}(\tau, 0)=\frac{\partial \theta_{\mathrm{e} 2}}{\partial Z}(\tau, 1)=\frac{\partial \theta_{\mathrm{l} 2}}{\partial Z}(\tau, 1)=0 \\
& \theta_{\mathrm{e} 1}(\tau, r)=\theta_{\mathrm{e} 2}(\tau, r), \quad \theta_{\mathrm{l} 1}(\tau, r)=\theta_{\mathrm{l} 2}(\tau, r) \\
& \frac{\partial \theta_{\mathrm{e} 1}}{\partial Z}(\tau, r)=k_{\mathrm{eR}} \frac{\partial \theta_{\mathrm{e} 2}}{\partial Z}(\tau, r), \quad \frac{\partial \theta_{\mathrm{l} 1}}{\partial Z}(\tau, r)=k_{\mathrm{lR}} \frac{\partial \theta_{\mathrm{l}}}{\partial Z}(\tau, r)
\end{aligned}
$$

In Eqs. (1-4), it is assumed that the conduction of heat by phonons is not neglected and that the incident laser energy is absorbed by both electron gas and solid lattice. Assuming that conduction of heat may be carried by phonons, in addition to electrons, is a justified assumption for metals containing large amount of impurities or for semiconducting materials, where it is known that energy may be diffused by both solid lattice and electron gas in these materials $[16,17]$. Depending on the nature of heating methods and the structure of materials, it is known that energy deposits into materials in different ways. For example, energy can deposit simultaneously on all energy carriers (e.g., electrons and phonons) through contact heating at surfaces, or selectively on a particular group of carriers by radiation heating. Radiation heating excites free/bound electrons in metals, but excites valence electrons or optical phonons in semiconductors. So it is necessary for each one of the energy equations of the 
parabolic two-step models to contain a source term to account for that part of incident radiation absorbed by its energy carriers which are electrons or phonons. Equation (5) assumes that the thin composite slab is thermaly insulated from its external boundaries. This is justified since the duration of the heating process is very short and as a result, the thermal losses from the slab are of insignificant effects.

Equations (1-4) are four coupled partial differential equations which are second order in space and first order in time. Two types of coupling exist among these equations. These are the direct coupling between Eqs. (1) and (2) and between Eqs. (3) and (4), and the indirect coupling through boundary conditions between Eqs. (1) and (2) from one side and (3) and (4) from the other side. Elimination the coupling between these equations yields four partial differential equations of higher orders contain mixed derivatives which are indirectly coupled through boundary conditions. The higher order and mixed derivative terms appear in the obtained equations raise the difficulty of solving such problems. However, in many applications, the coupling between the energy equations (1-4) may be eliminated without raising the order of the obtained partial differential equations and without the appearance of mixed derivative terms. These applications involve situations in which the incident thermal radiation interacts with materials having very large coupling factor between electron gas and solid lattice or situations in which the laser pulse duration is not too short. In these situations, the difference between the electron and lattice temperatures is very small, but not neglected, and may be normalized in the form of a very small perturbed quantity. These differences may be written as:

$\theta_{\mathrm{e} 1}(\tau, \xi)=\theta_{\mathrm{l} 1}(\tau, \xi)+\epsilon_{1} \Delta_{1}(\tau, \xi)$

$\theta_{\mathrm{e} 2}(\tau, \xi)=\theta_{\mathrm{l} 2}(\tau, \xi)+\epsilon_{2} \Delta_{2}(\tau, \xi)$

where $\Delta_{1}(\tau, \xi)$ and $\Delta_{2}(\tau, \xi)$ are functions of space and time, $\epsilon_{1}=1 / H_{1}$ and $\epsilon_{2}=1 / H_{5}$ are dimensionless small parameters. As the coupling factors $G_{1}$ and $G_{2}$ increase, $H_{1}$ and $H_{5}$ increase and $\epsilon_{1}$ and $\epsilon_{2}$ decrease. In the limit as $G_{1}$ and $G_{2}$ become very large, then $\theta_{\mathrm{e} 1}$ approaches $\theta_{11}$ and $\theta_{\mathrm{e} 2}$ approaches $\theta_{12}$ and then the macroscopic heat conduction models may be adopted. Consider, for example, the interaction of laser beam with a very thin lead $(\mathrm{Pb})$ film of $1 \times 10^{-6} \mathrm{~m}$ thickness. For lead, the thermal conductivity and coupling factor have values of $35 \mathrm{~W} / \mathrm{m} \mathrm{K}$ and $12.4 \times 10^{16} \mathrm{~W} / \mathrm{m}^{3} \mathrm{~K}$, respectively $[10,14]$. Under these conditions, $H_{1}=3500$ and as a result, $\epsilon\left(=1 / H_{1}\right)$ may be considered as a very small perturbed quantity. Examples of other metals having very large coupling factor (i.e., having very small perturbed parameter $\epsilon$ ) are Vanadium (V), Niobium (Nb), Titanium (Ti), etc. Now, eliminate $\theta_{\mathrm{e} 1}-\theta_{\mathrm{l1}}$ between Eqs. (1) and (2) and $\theta_{\mathrm{e} 2}-\theta_{\mathrm{l} 2}$ between Eqs. (3) and (4), and drop terms of order $\epsilon_{1}, \epsilon_{2}$ and higher, yields:

$$
\begin{aligned}
& \frac{\partial \theta_{\mathrm{e} 1}}{\partial \tau}=A_{1} \frac{\partial^{2} \theta_{\mathrm{e} 1}}{\partial Z^{2}}+\frac{F_{1}}{H_{1}} P_{\mathrm{e} 1}+\frac{\left(1-F_{1}\right) P_{11}}{H_{3}} \\
& \frac{\partial \theta_{\mathrm{e} 2}}{\partial \tau}=A_{2} \frac{\partial^{2} \theta_{\mathrm{e} 2}}{\partial Z^{2}}+\frac{F_{2}}{H_{5}} P_{\mathrm{e} 2}+\frac{\left(1-F_{2}\right) P_{12}}{H_{7}}
\end{aligned}
$$

$$
\begin{aligned}
& \Delta_{1}=-\frac{\partial \theta_{\mathrm{e} 1}}{\partial \tau}+\frac{\partial^{2} \theta_{\mathrm{e} 1}}{\partial Z^{2}}+F_{1} P_{\mathrm{e} 1} \\
& \Delta_{2}=-\frac{\partial \theta_{\mathrm{e} 2}}{\partial \tau}+H_{4} \frac{\partial^{2} \theta_{\mathrm{e} 2}}{\partial Z^{2}}+F_{2} P_{\mathrm{e} 2}
\end{aligned}
$$

where

$$
A_{1}=\frac{\left(1 / H_{1}\right)+\left(H_{2} / H_{3}\right)}{\left(1 / H_{1}\right)+\left(1 / H_{3}\right)}, \quad A_{2}=\frac{\left(H_{4} / H_{5}\right)+\left(H_{6} / H_{7}\right)}{\left(1 / H_{5}\right)+\left(1 / H_{7}\right)}
$$

Analytical solutions for Eqs. (8) and (9) are expressed in terms of Green's function in the form [18]:

$\theta_{\mathrm{e} 1}(\tau, Z)$

$$
\begin{aligned}
& =\left.\int_{Z^{\prime}=0}^{r} G_{11}\left(Z, \tau \mid Z^{\prime}, \tau^{*}\right)\right|_{\tau^{*}=0} \theta_{\mathrm{e} 1}\left(0, Z^{\prime}\right) \mathrm{d} Z^{\prime} \\
& +\left.\int_{Z^{\prime}=r}^{1} G_{12}\left(Z, \tau \mid Z^{\prime}, \tau^{*}\right)\right|_{\tau^{*}=0} \theta_{\mathrm{e} 2}\left(0, Z^{\prime}\right) \mathrm{d} Z^{\prime} \\
& +\int_{\tau^{*}=0}^{\tau} \mathrm{d} \tau^{*} \int_{Z^{\prime}=0}^{r} G_{11}\left(Z, \tau \mid Z^{\prime}, \tau^{*}\right) \\
& \times\left[\frac{F_{1}}{H_{1}} P_{\mathrm{e} 1}\left(\tau^{*}, Z^{\prime}\right)+\frac{\left(1-F_{1}\right)}{H_{3}} P_{11}\left(\tau^{*}, Z^{\prime}\right)\right] \mathrm{d} Z^{\prime} \\
& +\int_{\tau^{*}=0}^{\tau} \mathrm{d} \tau^{*} \int_{Z^{\prime}=r}^{1} G_{12}\left(Z, \tau \mid Z^{\prime}, \tau^{*}\right) \\
& \times\left[\frac{F_{2}}{H_{5}} P_{\mathrm{e} 2}\left(\tau^{*}, Z^{\prime}\right)+\frac{\left(1-F_{2}\right)}{H_{7}} P_{\mathrm{l} 2}\left(\tau^{*}, Z^{\prime}\right)\right] \mathrm{d} Z^{\prime} \\
& \theta_{\mathrm{e} 2}(\tau, Z) \\
& =\left.\int_{Z^{\prime}=0}^{r} G_{21}\left(Z, \tau \mid Z^{\prime}, \tau^{*}\right)\right|_{\tau^{*}=0} \theta_{\mathrm{e} 1}\left(0, Z^{\prime}\right) \mathrm{d} Z^{\prime} \\
& +\left.\int_{Z^{\prime}=r}^{1} G_{22}\left(Z, \tau \mid Z^{\prime}, \tau^{*}\right)\right|_{\tau^{*}=0} \theta_{\mathrm{e} 2}\left(0, Z^{\prime}\right) \mathrm{d} Z^{\prime} \\
& +\int_{\tau^{*}=0}^{\tau} \mathrm{d} \tau^{*} \int_{Z^{\prime}=0}^{r} G_{21}\left(Z, \tau \mid Z^{\prime}, \tau^{*}\right) \\
& \times\left[\frac{F_{1}}{H_{1}} P_{\mathrm{e} 1}\left(\tau^{*}, Z^{\prime}\right)+\frac{\left(1-F_{1}\right)}{H_{3}} P_{11}\left(\tau^{*}, Z^{\prime}\right)\right] \mathrm{d} Z^{\prime} \\
& +\int_{\tau^{*}=0}^{\tau} \mathrm{d} \tau^{*} \int_{Z^{\prime}=r}^{1} G_{22}\left(Z, \tau \mid Z^{\prime}, \tau^{*}\right) \\
& \times\left[\frac{F_{2}}{H_{5}} P_{\mathrm{e} 2}\left(\tau^{*}, Z^{\prime}\right)+\frac{\left(1-F_{2}\right)}{H_{7}} P_{12}\left(\tau^{*}, Z^{\prime}\right)\right] \mathrm{d} Z^{\prime}
\end{aligned}
$$

Here $G_{i j}\left(Z, \tau \mid Z^{\prime}, \tau^{*}\right)$ are the appropriate Green's functions which are given as:

$G_{i j}\left(Z, \tau \mid Z^{\prime}, \tau^{*}\right)=\sum_{n=1}^{\infty} \frac{\mathrm{e}^{-\beta_{n}^{2}\left(\tau-\tau^{*}\right)}}{N_{n}} C_{j} \Psi_{i n}(Z) \Psi_{j n}\left(Z^{\prime}\right)$ 
where $C_{1}=1 / A_{1}, C_{2}=k_{\mathrm{eR}} / A_{2}$, and $\Psi_{i n}$ s are the eigenfunctions obtained from the solution of the homogeneous version of Eqs. (8) and (9), as:

$$
\begin{aligned}
& \Psi_{1 n}=\cos \left(\frac{\beta_{n}}{\sqrt{A_{1}}} Z\right) \\
& \Psi_{2 n}=E_{1 n} \cos \left(\frac{\beta_{n}}{\sqrt{A_{2}}} Z\right)+E_{2 n} \sin \left(\frac{\beta_{n}}{\sqrt{A_{2}}} Z\right)
\end{aligned}
$$

where $E_{1 n}$ and $E_{2 n}$ are found as: and

$$
a=\frac{\beta_{n}}{\sqrt{A_{1}}}, \quad b=\frac{\beta_{n}}{\sqrt{A_{2}}}
$$

Also, $\Delta_{1}$ and $\Delta_{2}$ are obtained in terms of Eqs. (10), (11) and (18), (19), as:

$$
\begin{aligned}
& \Delta_{1}(\tau, Z) \\
& \quad=\sum_{n=1}^{\infty} \sqrt{A_{1}} \frac{C_{1}}{N_{n}} R \beta_{n} \mathrm{e}^{-\beta_{n}^{2}\left(\tau-\tau_{0}\right)} \sin \left(\frac{\beta_{n} r}{\sqrt{A_{1}}}\right) \cos \left(\frac{\beta_{n} Z}{\sqrt{A_{1}}}\right)
\end{aligned}
$$

$E_{1 n}=\frac{\cos \left(\beta_{n} r / \sqrt{A_{1}}\right) \cos \left(\beta_{n} / \sqrt{A_{2}}\right)}{\cos \left(\beta_{n} r / \sqrt{A_{2}}\right) \cos \left(\beta_{n} / \sqrt{A_{2}}\right)+\sin \left(\beta_{n} r / \sqrt{A_{2}}\right) \sin \left(\beta_{n} / \sqrt{A_{2}}\right)}$
$E_{2 n}=\frac{\cos \left(\beta_{n} r / \sqrt{A_{1}}\right) \sin \left(\beta_{n} / \sqrt{A_{2}}\right)}{\cos \left(\beta_{n} r / \sqrt{A_{2}}\right) \cos \left(\beta_{n} / \sqrt{A_{2}}\right)+\sin \left(\beta_{n} r / \sqrt{A_{2}}\right) \sin \left(\beta_{n} / \sqrt{A_{2}}\right)}$

and $\beta_{n}$ s are the roots of:

$$
\begin{aligned}
- & \frac{1}{\sqrt{A_{1}}} \sin \left(\frac{\beta_{n} r}{\sqrt{A_{1}}}\right) \\
& =k_{\mathrm{eR}}\left\{-\frac{E_{1 n}}{\sqrt{A_{2}}} \sin \left(\frac{\beta_{n} r}{\sqrt{A_{2}}}\right)+\frac{E_{2 n}}{\sqrt{A_{2}}} \cos \left(\frac{\beta_{n} r}{\sqrt{A_{2}}}\right)\right\}
\end{aligned}
$$

Now, no further processing on the solution can be conducted without specifying the nature of the heating source. Consider an impulsive energy source released at time $\tau=\tau_{0}$ and assume that all of the incident energy is absorbed completely by the electron gas of the first layer. As a result, $F_{1}=1$ and $P_{\mathrm{e} 2}=P_{12}=0$, and

$\frac{P_{\mathrm{e} 1}}{H_{1}}=R \delta\left(\tau-\tau_{0}\right)$

where $R=h_{2}^{2} Q_{\mathrm{el}} / k_{\mathrm{e} 1} H_{1} T_{\mathrm{i}}$, and $\delta$ is the Dirac's delta function. Such an energy source could model, for example, the application of a strong laser pulse which releases its energy through an absorbing medium encountered in the annealing of semiconductors [19]. The temperature distribution can be directly obtained by substituting Eq. (17) into the general solution as given in Eqs. (12) and (13), and therefore,

$$
\begin{aligned}
\theta_{\mathrm{e} 1}(\tau, Z)= & \sum_{n=1}^{\infty} \frac{\sqrt{A_{1}}}{\beta_{n}} \frac{C_{1}}{N_{n}} R \mathrm{e}^{-\beta_{n}^{2}\left(\tau-\tau_{0}\right)} \\
& \times \sin \left(\frac{\beta_{n} r}{\sqrt{A_{1}}}\right) \cos \left(\frac{\beta_{n} Z}{\sqrt{A_{1}}}\right)
\end{aligned}
$$

$$
\begin{aligned}
\theta_{\mathrm{e} 2}(\tau, Z) & \\
= & \sum_{n=1}^{\infty} \frac{\sqrt{A_{1}}}{\beta_{n}} \frac{C_{1}}{N_{n}} R \mathrm{e}^{-\beta_{n}^{2}\left(\tau-\tau_{0}\right)} \sin \left(\frac{\beta_{n} r}{\sqrt{A_{1}}}\right) \\
& \times\left\{E_{1 n} \cos \left(\frac{\beta_{n} Z}{\sqrt{A_{2}}}\right)+E_{2 n} \sin \left(\frac{\beta_{n} Z}{\sqrt{A_{2}}}\right)\right\}
\end{aligned}
$$

where

$$
\begin{aligned}
N_{n}= & C_{1}\left\{\frac{Z}{2}+\frac{\sin 2 a Z}{4 a}\right\}_{0}^{r}+C_{2}\left\{E_{1 n}^{2}\left(\frac{Z}{2}+\frac{\sin 2 b Z}{4 b}\right)\right. \\
& \left.+E_{2 n}^{2}\left(\frac{Z}{2}-\frac{\sin 2 b Z}{4 b}\right)+2 E_{1 n} E_{2 n}\left(\frac{\sin ^{2} b Z}{2 b}\right)\right\}_{r}^{1}
\end{aligned}
$$

$$
\begin{aligned}
&-\sum_{n=1}^{\infty} \frac{C_{1}}{N_{n}} \frac{R}{\sqrt{A_{1}}} \beta_{n} \mathrm{e}^{-\beta_{n}^{2}\left(\tau-\tau_{0}\right)} \sin \left(\frac{\beta_{n} r}{\sqrt{A_{1}}}\right) \\
& \times \cos \left(\frac{\beta_{n} Z}{\sqrt{A_{1}}}\right)+R \delta\left(\tau-\tau_{o}\right) \\
& \Delta_{2}(\tau, Z)= \sum_{n=1}^{\infty} \sqrt{A_{1}} \frac{C_{1}}{N_{n}} R \beta_{n} \mathrm{e}^{-\beta_{n}^{2}\left(\tau-\tau_{0}\right)} \sin \left(\frac{\beta_{n} r}{\sqrt{A_{1}}}\right) \\
& \times\left\{E_{1 n} \cos \left(\frac{\beta_{n} Z}{\sqrt{A_{2}}}\right)+E_{2 n} \sin \left(\frac{\beta_{n} Z}{\sqrt{A_{2}}}\right)\right\} \\
&-\sum_{n=1}^{\infty} H_{4} \sqrt{A_{1}} \frac{C_{1}}{N_{n}} \frac{R}{A_{2}} \beta_{n} \mathrm{e}^{-\beta_{n}^{2}\left(\tau-\tau_{0}\right)} \sin \left(\frac{\beta_{n} r}{\sqrt{A_{1}}}\right) \\
& \times\left\{E_{1 n} \cos \left(\frac{\beta_{n} Z}{\sqrt{A_{2}}}\right)+E_{2 n} \sin \left(\frac{\beta_{n} Z}{\sqrt{A_{2}}}\right)\right\}(21)
\end{aligned}
$$

To verify the validity of the obtained results, the difference function $\Delta_{1}$ in the first layer of a two-layer composite film is compared with the difference function in a single layer. The comparison is conducted in a two-layer composite film having $r=0.98$. This implies that the second layer is of very small thickness. Figure 1 shows the comparison between the transient behavior of the two difference functions. The results obtained using the following parameters:

$H_{1}=1000, \quad H_{2}=0.01, \quad H_{3}=1000, \quad H_{4}=0.01$,

$H_{5}=1000, \quad H_{6}=0.01, \quad H_{7}=1000, \quad \tau_{0}=3$

$F_{1}=1, \quad P_{\mathrm{e} 2}=P_{12}=0, \quad r=0.98, \quad R=1$

$k_{\mathrm{eR}}=1, \quad k_{\mathrm{lR}}=1, \quad Z=0.5$

It is clear from Fig. 1 that the difference function in the first layer of the composite film is very close to the difference function of a single layer. This is because of the very small thickness of the second layer. As a result, the first layer in the composite film behaves as if the second layer is not exist. Also, it is clear from the same figure that both difference functions go to zero as time increases. As time proceeds, the electron gas exchange its energy with the solid lattice and the difference between the two temperatures decreases. In the limit, both the 


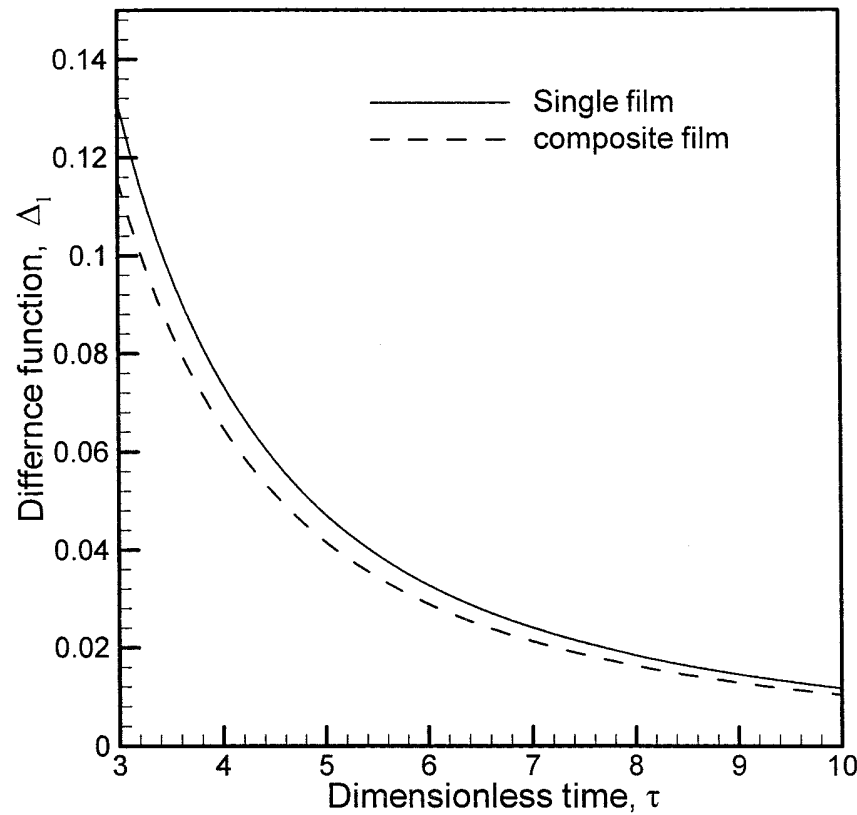

Fig. 1. The difference function at the center of the single film and at the center of the first layer in the composite film

electron gas and the solid lattice attain the same temperature. The figure shows that the difference function in the composite film is slightly lower than that in the single layer. The electron gas in the first layer of the composite film losses small part of its energy to the second thin domain in addition to the major part lost to the solid lattice of the first domain. As a result, the difference function in the composite film is less than that in the single film.

Also, consider another heating source term in which the spatial distribution of the laser intensity is assumed to decay exponentially in the first layer and the temporal shape of the laser pulse is assumed to be Gaussian with a full width at half maximum (FWHM) pulse duration $\tau_{\mathrm{p}}$, peaking at time $\tau_{\mathrm{p}}$. This source term is given as [11]:

$P_{\mathrm{e} 1}=H_{1} M \mathrm{e}^{-\alpha h_{2} Z} \mathrm{e}^{-2.77 \tau^{2} / \tau_{\mathrm{p}}^{2}} \mathrm{e}^{11.08 \tau / \tau_{\mathrm{p}}}$

and $P_{\mathrm{e} 2}=P_{11}=P_{12}=0$, and $M=\left(h_{2}^{2} / k_{\mathrm{e} 1} T_{\mathrm{i}}\right)(1-\rho)$ $I \alpha \mathrm{e}^{-11.08}$. Insert Eq. (22) into (12), (13) and integrate to yield:

$$
\begin{aligned}
\theta_{\mathrm{e} 1}(\tau, Z)= & \sum_{n=1}^{\infty} \mathrm{e}^{-\beta_{n}^{2} \tau} \cos \left(\frac{\beta_{n} Z}{\sqrt{A_{1}}}\right) \Lambda_{1} \\
\times & {\left[\operatorname{erf}\left(-c \sqrt{-\frac{1}{4 d}}+\frac{\tau}{2 \sqrt{-\frac{1}{4 d}}}\right)\right.} \\
& \left.-\operatorname{erf}\left(-c \sqrt{-\frac{1}{4 d}}\right)\right]
\end{aligned}
$$

$\theta_{\mathrm{e} 2}(\tau, Z)$

$$
=\sum_{n=1}^{\infty} \mathrm{e}^{-\beta_{n}^{2} \tau}\left\{E_{1 n} \cos \left(\frac{\beta_{n} Z}{\sqrt{A_{2}}}\right)+E_{2 n} \sin \left(\frac{\beta_{n} Z}{\sqrt{A_{2}}}\right)\right\} \Lambda_{1}
$$

$$
\begin{gathered}
\times\left[\operatorname{erf}\left(-c \sqrt{-\frac{1}{4 d}}+\frac{\tau}{2 \sqrt{-\frac{1}{4 d}}}\right)\right. \\
\left.-\operatorname{erf}\left(-c \sqrt{-\frac{1}{4 d}}\right)\right]
\end{gathered}
$$

where

$$
\begin{aligned}
& \Lambda_{1}=\frac{C_{1}}{N_{n}} M\left\{\mathrm{e}^{a Z} \frac{a \cos b Z+b \sin b Z}{a^{2}+b^{2}}\right\}_{0}^{r} \sqrt{-\frac{\pi}{4 d}} \mathrm{e}^{-c^{2} / 4 d}, \\
& a=-\alpha h_{2}, \quad b=\frac{\beta_{n}}{\sqrt{A_{1}}}, \quad c=\beta_{n}^{2}+\frac{11.08}{\tau_{\mathrm{p}}}, \quad d=-\frac{2.77}{\tau_{\mathrm{p}}^{2}}
\end{aligned}
$$

and $\Delta_{1}$ and $\Delta_{2}$ are obtained from Eqs. (10), (11) and (23), (24), with:

$$
\begin{aligned}
& \frac{\partial \theta_{\mathrm{e} 1}}{\partial \tau}=\sum_{n=1}^{\infty} \mathrm{e}^{-\beta_{n}^{2} \tau} \cos \left(\frac{\beta_{n} Z}{\sqrt{A_{1}}}\right) \Lambda_{1} \frac{2}{\sqrt{\pi}} \\
& \times\left\{\mathrm{e}^{-[-c \sqrt{-1 / 4 d}+\tau / 2 \sqrt{-1 / 4 d}]^{2}}-\mathrm{e}^{-[-c \sqrt{-1 / 4 d}]^{2}}\right\} \\
& -\sum_{n=1}^{\infty} \beta_{n}^{2} \mathrm{e}^{-\beta_{n}^{2} \tau} \cos \left(\frac{\beta_{n} Z}{\sqrt{A_{1}}}\right) \Lambda_{1} \\
& \times\left[\operatorname{erf}\left(-c \sqrt{-\frac{1}{4 d}}+\frac{\tau}{2 \sqrt{-1 / 4 d}}\right)\right. \\
& \left.-\operatorname{erf}\left(-c \sqrt{-\frac{1}{4 d}}\right)\right]
\end{aligned}
$$

$\frac{\partial \theta_{\mathrm{e} 2}}{\partial \tau}$

$$
\begin{aligned}
& =\sum_{n=1}^{\infty} \mathrm{e}^{-\beta_{n}^{2} \tau}\left[E_{1 n} \cos \left(\frac{\beta_{n} Z}{\sqrt{A_{2}}}\right)+E_{2 n} \sin \left(\frac{\beta_{n} Z}{\sqrt{A_{2}}}\right)\right] \\
& \times \Lambda_{1} \frac{2}{\sqrt{\pi}}\left\{\mathrm{e}^{-[-c \sqrt{-1 / 4 d}+\tau / 2 \sqrt{-1 / 4 d}]^{2}}-\mathrm{e}^{-[-c \sqrt{-1 / 4 d}]^{2}}\right\} \\
& -\sum_{n=1}^{\infty} \beta_{n}^{2} \mathrm{e}^{-\beta_{n}^{2} \tau}\left[E_{1 n} \cos \left(\frac{\beta_{n} Z}{\sqrt{A_{2}}}\right)+E_{2 n} \sin \left(\frac{\beta_{n} Z}{\sqrt{A_{2}}}\right)\right] \\
& \times \Lambda_{1}\left[\operatorname{erf}\left(-c \sqrt{-\frac{1}{4 d}}+\frac{\tau}{2 \sqrt{-1 / 4 d}}\right)\right. \\
& \left.-\operatorname{erf}\left(-c \sqrt{-\frac{1}{4 d}}\right)\right] \\
& \frac{\partial^{2} \theta_{\mathrm{e} 1}}{\partial Z^{2}}=-\sum_{n=1}^{\infty} \mathrm{e}^{-\beta_{n}^{2} \tau} \frac{\beta_{n}^{2}}{A_{1}} \cos \left(\frac{\beta_{n} Z}{\sqrt{A_{1}}}\right) \\
& \times \Lambda_{1}\left[\operatorname{erf}\left(-c \sqrt{-\frac{1}{4 d}}+\frac{\tau}{2 \sqrt{-1 / 4 d}}\right)\right. \\
& \left.-\operatorname{erf}\left(-c \sqrt{-\frac{1}{4 d}}\right)\right]
\end{aligned}
$$




$$
\begin{aligned}
& \frac{\partial^{2} \theta_{\mathrm{e} 2}}{\partial Z^{2}} \\
& =-\sum_{n=1}^{\infty} \mathrm{e}^{-\beta_{n}^{2} \tau} \frac{\beta_{n}^{2}}{A_{2}}\left\{E_{1 n} \cos \left(\frac{\beta_{n} Z}{\sqrt{A_{2}}}\right)+E_{2 n} \sin \left(\frac{\beta_{n} Z}{\sqrt{A_{2}}}\right)\right\} \\
& \times \Lambda_{1}\left[\operatorname{erf}\left(-c \sqrt{-\frac{1}{4 d}}+\frac{\tau}{2 \sqrt{-1 / 4 d}}\right)\right. \\
& \left.\quad-\operatorname{erf}\left(-c \sqrt{-\frac{1}{4 d}}\right)\right]
\end{aligned}
$$

\section{3}

\section{Conclusion}

Using a simple perturbation technique, an analytical investigation is presented for the heat transfer mechanisms during ultrafast laser heating of a perfectly contacted twolayer composite thin slab from a microscopic point of view. Analytical expressions for the electron gas and solid lattice temperature distributions in both layers are obtained. The thermal behavior of the slab is described under the effect of heating sources of two types. The first is impulsive in time and uniform is space and the second is Gaussian in time and exponentially decaying is space. The microscopic parabolic two-step model is adopted to describe the behavior of the composite slab. The proposed perturbation technique is used when the normalized temperature difference between the solid lattice and the electron gas is relatively a small perturbed quantity.

\section{References}

1. Boyd IW (1989) Laser Processing of Thin Films and Microstructures. Springer, New York

2. Chryssolouris G (1991) Laser Machining, Theory and Practice. Springer, New York

3. Fujimoto JG; Liu JM; Ippen EP (1984) Femtosecond laser interaction with metallic tungsten and non-equilibrium electron and lattice temperature. Phys Rev Lett 53: 18371840

4. Elsayed-Ali HE (1991) Femtosecond thermoreflectivity and thermotransmissivity of polycrystalline and single-crystalline gold films. Phys Rev B43: 4488-4491

5. Kaganov MI; Lifshitz IM; Tanatarov LV (1957) Relaxation between electrons and crystalline lattices. Sov Phys JETP 4: 173-178

6. Eesley GL (1983) Observation of non-equilibrium electron heating in copper. Phys Rev Lett 51: 2140-2143

7. Kim WS; Hector LG; Ozisik MN (1990) Hyperbolic heat conduction due to axisymmetric continuous or pulsed surface heat sources. J Appl Phys 68: 5478-5485

8. Chen H-T; Lin J-Y (1994) Analysis of two-dimensional hyperbolic heat conduction problems. Int J Heat Mass Transfer 37(1): 153-164

9. Maxwell JC (1867) On the dynamic theory of gases. Philos Trans Soc London 157: 49-88

10. Qiu TQ; Tien CL (1992) Short-pulse laser heating on metals. Int J Heat Mass Transfer 35(3): 719-726

11. Qiu TQ; Tien CL (1993) Heat transfer mechanism during short-pulse laser heating of metals. J Heat Transfer 115: 835841

12. Tzou DY; Ozisik MN; Chiffelle RJ (1994) The lattice temperature in the microscopic two-step model. J Heat Transfer 116: $1034-1038$

13. Tzou DY (1995) A unified field approach for heat conduction from macro- to micro-scales. J Heat Transfer 117: 8-16

14. Tzou DY (1995) The generalized lagging response in smallscale and high-rate heating. Int J Heat Mass Transfer 38(17): 3231-3240

15. Al-Nimr MA; Masoud S (1997) Nonequilibrium laser heating of metal films. J Heat Transfer 119: 188-190

16. Al-Nimr MA (1997) Heat transfer mechanisms during shortduration laser heating of thin metal films. Int J Thermophys 18(5): 1257-1268

17. Blakemore JS (1974) Solid State Physics, 2nd edn., Ch. 3, Cambridge University Press, London

18. Ozisik MN (1993) Heat Conduction, 2nd edn., John Wiley, Ch. 6, pp. 214-256

19. Appleton BR; Cellar GK (eds.) (1982) Laser and ElectronBeam Interaction with Solids. Elsevier Science, New York 